\title{
La dimensión social del movimiento estudiantil de Córdoba en 1918
}

\author{
The social dimension of the student's movement at Cordoba in 1918
}

Andrés Donoso Romo* \& Rafael Contreras Mühlenbrock ${ }^{* *}$

\begin{abstract}
Resumen: El artículo demuestra que el movimiento estudiantil de Córdoba en 1918 fue mucho más que cogobierno, también fue la expresión de una nueva sensibilidad social que impulsaba a los jóvenes a involucrarse en los destinos de la sociedad. Para asir este objetivo se esbozará el entramado cultural desde donde surge el movimiento, se describirán las fases e hitos que lo caracterizaron, se estudiarán las principales demandas que se enarbolaron y se reparará en las diferentes pistas que informan sobre esta nueva sensibilidad.
\end{abstract}

Palabras clave: Movimientos estudiantiles, Autonomía universitaria, Universidades populares, Argentina, América Latina.

\begin{abstract}
The article allow to understand the Cordoba's student movement in 1918, as one expressions of the new social sensitivity, which the Latin-American students will have since then. This will push them to look for a larger role solving society issues. To achieve this goal, the cultural links where the movement comes from will be outlined, stages and milestones that characterized it will be described, the main demands raised will be studied and the different clues that inform about this new social sensitivity will be repaired.
\end{abstract}

Key words: Students' movement, university autonomy, popular universities, Argentina, Latin America.

Recibido: 25 noviembre 2016

Aceptado: 14 marzo 2017

\footnotetext{
* Chileno, autor principal. Doctor en Integración de América Latina por la Universidade de São Paulo. Investigador del Centro de Estudios Avanzados de la Universidad de Playa Ancha. El artículo informa resultados del estudio "Movimientos estudiantiles universitarios en América Latina (1918-2011): aproximación histórica a los papeles atribuidos a la educación en la transformación social", CONICYT/FONDECYT, Concurso de Iniciación, $\mathrm{N}^{\circ} 11140250$, 2014-2017. Correo electrónico: andres.donoso@upla.cl

** Chileno. Licenciado en Antropología Social por la Universidad de Chile. Documentalista e investigador independiente en temas de cultura popular. Correo electrónico: rafa_acm@ yahoo.com
} 


\section{Introducción}

En octubre de 1918 el estudiantado universitario cordobés, luego de un año de movilizaciones, consiguió una conquista que lo inscribió entre los grandes protagonistas de la historia contemporánea de América Latina ${ }^{1}$. ¿Qué obtuvo? Plena participación en el gobierno universitario. Independientemente de lo transitoria que haya sido esta victoria, pues en los años siguientes fue afectada por los vaivenes propios de toda lucha por la hegemonía, no hay duda que para una porción importante del estudiantado regional ella se convirtió en un faro que al mismo tiempo que demarcaba sendas, apuntaba desafíos. Para los estudiosos, en tanto, este movimiento estudiantil universitario -en adelante movimiento estudiantil o solo movimiento- tendió a adquirir un carácter fundacional, en el sentido que se lo entendió como el primero en alcanzar resonancia continental y, más importante aún, se lo asumió como un antecedente ineludible para comprender a la universidad latinoamericana actual. Con todo, hay un aspecto de este movimiento que no ha sido objeto de reflexiones sistemáticas pese a ser advertido tempranamente por algunos analistas, a saber, el que fuera una de las primeras veces en que el estudiantado expresó la inquietud por lograr una vinculación más estrecha entre la universidad y la sociedad ${ }^{2}$.

Pero así como es justo subrayar la originalidad del movimiento cordobés, también es necesario destacar que éste se insertaba en un ciclo de movilizaciones estudiantiles más amplio, de alcance latinoamericano, que se remontó hasta los albores del siglo XX y que se

\footnotetext{
${ }^{1}$ Se asume, como lo hacen, entre otros, Pablo González Casanova (1985) y Roberto Fernández Retamar, que la época contemporánea de América Latina comienza a fines del siglo XIX e inicios del siglo XX. Deslinde que se establece considerando, principalmente, los cambios en los modos de producción y de asentamiento que desde entonces comienza a experimentar la población. Véase, Pablo González Casanova, Imperialismo y liberación: una introducción a la historia contemporánea de América Latina, Ciudad de México, Siglo XXI Editores, 1985, 11; y Roberto Fernández Retamar, Pensamiento de Nuestra América, Buenos Aires, Consejo Latinoamericano de Ciencias Sociales, 2006, 39.

2 Junto al historiador Pablo Requena se advierte que tempranamente algunos analistas repararon en esta dimensión, como los casos de Julio V. González, José Carlos Mariátegui y Gabriel Del Mazo. Consultar Pablo Requena, Han pasado este año cosas estupendas. Ha florecido una nueva generación: Deodoro Roca y la construcción del imaginario reformista (Córdoba 1915-1942), Córdoba: Tesis Universidad Nacional de Córdoba, 2007, 8. Quien desee cotejar directamente estas fuentes ver Julio V. González, Significado de la Reforma Universitaria, Dardo Cúneo (comp.) La reforma Universitaria (1918-1930), Caracas, Biblioteca Ayacucho, 1988 [1923], 187, 192, 195 y 204; José Carlos Mariátegui, La Reforma Universitaria, Emir Sader y otros (comps.) La Reforma Universitaria: desafíos y perspectivas noventa años después, Buenos Aires, Consejo Latinoamericano de Ciencias Sociales, 2008 [1928], 208; y Gabriel del Mazo (comp.) La Reforma Universitaria. Tomo I: el movimiento argentino, Lima, Universidad Nacional Mayor de San Marcos, 1967, 88-90. Un estudio hoy clásico sobre los movimientos estudiantiles en América Latina también aborda este aspecto, aunque desde una perspectiva eminentemente sociológica, revisar Juan Carlos Portantiero, Estudiantes y política en América Latina: el proceso de la reforma universitaria (1918-1930), Ciudad de México, Siglo XXI Editores, 1987 [1978], 13. En años recientes otros autores también han identificado esta preocupación, como Nicolás Arata y Marcelo Mariño, La educación en la Argentina: una historia en 12 lecciones, Buenos Aires, Novedades Educativas, 2013, 177, y Marco Braguetto, El 'Grito de Córdoba' como hito histórico en disputa ideológica, Revista Izquierdas, $\mathrm{n}^{\circ}$ 15, Santiago de Chile, 2013, 93. Quien desee aproximarse a otro caso de principios del siglo XX donde esta nueva mentalidad se manifiesta consistentemente acudir a Fabio Moraga, Muchachos casi silvestres: la Federación de Estudiantes y el movimiento estudiantil chileno, 1906-1936, Santiago de Chile, Universidad de Chile, 2007, 207-219.
} 
proyectó hasta mediados del mismo siglo ${ }^{3}$. Encuadre que permite entender, por un lado, que el levantamiento cordobés fue la expresión de procesos más vastos que maduraban en toda América Latina ${ }^{4}$-comprensión a contrapelo de aquellas que lo entienden como un fenómeno particular que gozó de proyección continental- y, por otro lado, permite comprender que muchas de sus particularidades pudieron expresarse, precisamente, porque sus protagonistas formaban parte del entramado estudiantil, universitario y cultural que surgía en la región como respuesta a las transformaciones socioproductivas que desde hace algunas décadas se venían verificando.

A diferencia de las explicaciones más usuales que han dado los especialistas sobre el movimiento cordobés, las que han tendido a comprenderlo como un resultado del particular vínculo generacional que unía al estudiantado local, también como un desdoblamiento de las disputas entre seculares y clericales que se sucedían en la Argentina desde mediados del siglo XIX y, asimismo, como una consecuencia más del conjunto de transformaciones que experimentaba la política nacional e internacional en ese entonces ${ }^{5}$. Aquí se reconocerá la idoneidad de estos abordajes, pues todos identifican una parte de las razones que estaban detrás de esta movilización, pero se privilegiará otro acercamiento que releve su contexto social. Aproximación que permitirá, entre otras cosas, apreciar las articulaciones y similitudes que este fenómeno presentaba con otras movilizaciones estudiantiles que en esos mismos años se sucedían en América Latina.

En términos metodológicos el estudio desde donde se desprenden los resultados que aquí se exponen fue de carácter exploratorio y descansó en un proceso sistemático de análisis de contenido de las principales fuentes primarias y secundarias sobre el movimiento cordobés. Entre las primeras se cuentan algunos de los documentos elaborados por sus protagonistas, como periódicos, manifiestos o actas de congresos, y entre las segundas las reflexiones que a posteriori publicaron algunos observadores privilegiados y

\footnotetext{
${ }^{3}$ Respecto a sus precedentes no pocos/as investigadores/as reparan en que una parte importante de las exigencias que movilizaba a la juventud argentina habían sido discutidas diez años antes en el Primer Encuentro de Estudiantes Americanos realizado en 1908 en Montevideo, instancia que congregó a más de un centenar de estudiantes provenientes de Argentina, Bolivia, Brasil, Chile, Paraguay, Perú y Uruguay. Véase, por ejemplo, Hugo Biagini, La contracultura juvenil: de la emancipación a los indignados, Buenos Aires, Capital Intelectual, 2012, 43-52. Sobre sus desdoblamientos, en tanto, se apunta que el petitorio cordobés seguirá siendo la espina dorsal de las demandas estudiantiles hasta mediados del siglo XX, como se comprueba en las movilizaciones uruguayas de 1958, las brasileñas de 1962 e, inclusive, las mexicanas de comienzos de la década de los setenta. Véase, respectivamente, Vania Markarian, María Eugenia Jung \& Isabel Wschebor, 1958 El cogobierno autonómico, Montevideo, Archivo General de la Universidad de La República, 2008b, 99-102; Arthur Jose Poerner, O poder jovem: história da participação política dos estudantes brasileiros, São Paulo, Centro de Memória da Juventude, 1995 [1968], 182-183; y José Rivas Ontiveros, La izquierda estudiantil en la UNAM: organizaciones, movilizaciones y liderazgos (1958-1972), Ciudad de México, Editorial Miguel Ángel Porrúa/UNAM, 2007, 687.

${ }^{4}$ Véase Moraga, op. cit., 213; Antenor Orrego, ¿Cuál es la cultura que creará América? Dardo Cúneo (comp.) La Reforma Universitaria (1918-1930), Caracas, Biblioteca Ayacucho, 1988 [1928], 257; y Carlos Tünnermann, Noventa años de la Reforma Universitaria de Córdoba (1918-2008), Buenos Aires, Consejo Latinoamericano de Ciencias Sociales, 2008, 71.

${ }^{5}$ Véanse, entre otros textos, María Caldelari y Patricia Funes, Escenas reformistas: la Reforma Universitaria 1918-1930, Buenos Aires, EUDEBA, 1998, 10-11; Roberto Ferrero, Historia crítica del movimiento estudiantil de Córdoba, Tomo 1 (1918-1943), Córdoba, Alción, 1999, 11; y Tünnermann, op. cit., 39-41.
} 
también parte sustancial de las contribuciones que desde distintos campos del conocimiento se han propuesto esclarecer este fenómeno. Cabe apuntar, a su vez, que para seleccionar las obras incorporadas al análisis y para clarificar algunos de los puntos en ellas trabajados, la investigación se apoyó en entrevistas semiestructuradas a pares investigadores ${ }^{6}$.

Para prevenir malos entendidos se puntualiza que la noción de movimiento estudiantil utilizada, desprendida de una definición provista por Marcela Pronko, lo entiende como ese conjunto de acciones, más o menos planificadas, que el estudiantado realiza con miras a transformar aspectos que considera perjudiciales para sí o su sociedad ${ }^{7}$. Delimitación que autoriza a comprender que los movimientos que han tenido mayor alcance, proyección o magnitud, han sido aquellos que identifican problemas capaces de interpelar a diversos sectores de la sociedad. Dicho con otras palabras, la fortaleza de un movimiento estaría dada tanto por el grado en que sus demandas reflejan problemáticas universitarias, educacionales y sociales, como por la resonancia que adquieren sus acciones en las discusiones intelectuales, en las agendas políticas y/o en el sentido común de la población. Conjunto de premisas que permiten sostener que adentrarse en el estudio de los movimientos estudiantiles contemporáneos es, también, explorar los desafíos que una parte sustancial de los sectores medios de la sociedad identifican como ineludibles.

Para evitar confusiones también se subraya que el artículo no se propone realizar una caracterización exhaustiva del movimiento de Córdoba, tampoco busca presentar nuevas fuentes sobre el mismo, ni pretende ser un compendio de las voces que en ese entonces le dieron vida. Más bien persigue, con vocación interdisciplinaria, identificar aquellos elementos que permiten dar consistencia a una línea de interpretación que ha sido escasamente relevada en la literatura especializada, aquella que ve al movimiento estudiantil cordobés también como una expresión consistente del cambio de mentalidad que desde entonces comienza experimentar el estudiantado latinoamericano. Un ejercicio que permitirá acercarnos, entre otros aspectos, a la respuesta que entonces se estaba dando a una interrogante que todavía hoy sigue vigente, ésta es, ¿Cuál sería el papel que podría desempeñar la educación/universidad en la transformación de la sociedad? Entendiendo por "transformación" a ese conjunto de cambios, progresos o mejoras que procuran contribuir a cimentar colectividades justas o igualitarias.

Para dar cuenta de esta interrogante a esta introducción le sucederán cinco secciones. La primera describirá en sus trazos fundamentales el contexto histórico donde se enmarcó el movimiento. La segunda caracterizará las principales fases e hitos que tuvo este fenómeno. La tercera profundizará en las principales demandas que levantaron los estudiantes con énfasis en las que permiten aprehender esta nueva sensibilidad. La cuarta identificará algunas pistas complementarias que ayudan a apreciar mejor esta emergente preocupación social entre el estudiantado. Y la quinta, a modo de conclusión, reconstruirá

\footnotetext{
${ }^{6}$ Los entrevistados, a quienes se agradece profundamente, fueron: Nicolás Arata, Hugo Biagini, Pablo Buchbinder, Victoria Chabrando, Vania Markarian, Renate Marsiske, Fabio Moraga, Javier Moyano, Pablo Requena y Horacio Sanguinetti.

${ }^{7}$ Véase Marcela Pronko, Procesos institucionales y estructuración del movimiento estudiantil. Universidad de Luján (1979-1990), Renate Marsiske (coord.) Movimientos estudiantiles en la historia de América Latina, volumen II, Ciudad de México, CESU/UNAM/Plaza y Valdés Editores, 1999, 241-242.
} 
la línea argumental expuesta y subrayará la importancia de observar integralmente a este movimiento que, por estos días, conmemora sus primeros cien años de historia.

\section{El movimiento cordobés en su contexto}

La Argentina de fines del siglo XIX y comienzos del XX, gracias a las transformaciones económicas que trajeron consigo los inicios de la mecanización, tecnificación e industrialización de los procesos productivos — agrícolas y ganaderos principalmente-, fue una de las sociedades más prósperas de la región. Una bonanza económica que debe ser puesta inmediatamente entre comillas debido a que, como informan infinidad de fuentes, ella coexistía con un sinfín de problemáticas sociales asociadas a la precariedad laboral y a las duras condiciones de vida que experimentaban los sectores populares. Se debe considerar, a su vez, que producto de los trastornos productivos y comerciales provocados por la Primera Guerra Mundial todas estas contradicciones se agudizaron y con ellas proliferaron la cesantía, el hambre y la violencia ${ }^{8}$. Antecedentes que ayudan a comprender por qué los principales estallidos sociales de estas décadas -la "Semana Trágica", las refriegas de "La Forestal" o la "Patagonia Rebelde"-, se produjeron durante el transcurso de este conflicto bélico o en los años inmediatamente posteriores. Siendo en este escenario que en 1917 se inició en Córdoba un ciclo de movilizaciones que solo concluirá en 1922, fenómeno en el cual participaron, entre otros actores, estudiantes universitarios, funcionarios municipales y ferroviarios ${ }^{9}$.

Pero aunque Córdoba participara de las dinámicas sociales y económicas que se experimentaban en el resto del país, se debe considerar que su mediterraneidad hacía que no se envolviera en ellas con la misma intensidad que presentaban las zonas portuarias, en especial las ubicadas en los márgenes del Río de La Plata. Condición fronteriza que explica, a su vez, que ahí convivieran con un ímpetu especial los idearios modernos y los tradicionales. Una combinación que, a la larga, terminará imprimiéndole a la provincia el sello de ser una de las más conservadoras entre las más poderosas del país. De hecho, no son pocos los analistas que recuerdan que en la cerrada disputa que desde mediados del siglo XIX se sucedía entre seculares y clericales - contienda que conoció varios momentos álgidos hasta bien entrado el siglo XX-, Córdoba era uno de los principales bastiones del clericalismo $^{10}$. Cúmulo de antecedentes que permite comprender, a su vez, por qué la

\footnotetext{
${ }^{8}$ Alejandro Cattaruzza, Historia de la Argentina 1916-1955, Buenos Aires, Siglo XXI Editores, 2012, 91112; y Eduardo Zimmermann, Los liberales reformistas. La cuestión social en la Argentina, 1890-1916, Buenos Aires, Sudamericana-Universidad de San Andrés, 1995, 11-14.

${ }^{9}$ Victoria Chabrando, El mundo ha vuelto a ponerse en movimiento en busca de nuevas fórmulas: la Reforma Universitaria en el ciclo de protestas cordobés, Córdoba, Tesis Universidad Nacional de Córdoba, 2010, 117; y María Vera de Flasch, Reformas, contrarreformas y movimientos estudiantiles en la Universidad de Córdoba (1870-1936), Renate Marsiske (coord.) Movimientos estudiantiles en la historia de América Latina, volumen III, Ciudad de México, CESU/UNAM/Plaza y Valdés Editores, 2006, 34.

${ }^{10}$ Véase Javier Moyano, Régimen oligárquico y transformación del sistema político: el caso de los grupos gobernantes en la provincia argentina de Córdoba, 1890-1930, Ciudad de México, Tesis El Colegio de México, 2006, 80-81; Juan Carlos Tedesco, Educación y sociedad en la Argentina (1880-1945), Buenos Aires, Siglo XXI Editores, 2003, 124.
} 
Universidad de Córdoba tenía la impronta de ser una de las más conservadoras del país. Fama obtenida por los contenidos que se impartían, también en los que se omitían, y por la sumatoria de una infinidad de detalles como, por ejemplo, el que los juramentos de grado se hicieran sobre los evangelios, que se enseñara derecho canónico y "deberes para con los siervos", que existiera una imagen sagrada a la cual todos debían reverenciar y/o que una fábrica de velas de los jesuitas funcionara dentro de sus instalaciones ${ }^{11}$.

Se debe considerar, a su vez, que fue en estos años que el estudiantado universitario comenzó a aumentar en forma sostenida. Dinámica que alcanzará su cénit a mediados del siglo XX y que obedecía, entre otras razones, a que una porción cada vez más significativa de los emergentes sectores medios de la población estuvo en condiciones de liberar a los jóvenes de las tareas productivas para mantenerlos más tiempo en los quehaceres formativos. Por esto, si en 1901 la matrícula universitaria en Córdoba no alcanzaba a las trescientas personas, a principios de la década de 1920 ella se empinaba por sobre las dos mil. Tendencia que se replicaba en el resto del país pues entre los años 1900 y 1910 el estudiantado universitario aumentó al doble y, si se consideran como parámetros los años de 1906 y 1920, éste se incrementó en seis veces ${ }^{12}$.

Y fue precisamente producto de estas dinámicas descritas que la juventud universitaria del país empieza a modificar sus concepciones sobre el mundo, la educación y el papel que debía asumir dentro de la universidad. Cambios que se insertaban, ciertamente, en los procesos culturales que se experimentaban en la sociedad, aquellos que se hacían patentes cuando en la cosmovisión el teocentrismo iba siendo desplazado por el antropocentrismo - proceso que no estuvo, ni está, exento de conflictos- y en la educación la escolástica iba siendo relegada por el empirismo ${ }^{13}$. Siendo estas transformaciones también las que permiten comprender que la juventud estudiosa, en sintonía con los postulados de José Enrique Rodó, José Ortega y Gasset o José Ingenieros, asumiera posiciones cada vez más activas en lo que se refiere a la construcción y uso del conocimiento. Cambios que le permitirán ir comprendiéndose como parte de la intelectualidad $\mathrm{y}$, en dicha condición, ir exigiendo, como en Córdoba, una mayor horizontalidad en su trato con el profesorado y con las autoridades universitarias. Lo que

11 Véase César Tcach, Movimiento estudiantil e intelectualidad reformista en Argentina (1918-1946), Cuadernos de Historia, 37, Santiago de Chile, 2012, 136; Portantiero, op. cit., 30-31, y Vera de Flasch, op. cit., 32.

${ }^{12}$ Interpretación en línea con la sostenida por Osvaldo Graciano, Entre la torre de marfil y el compromiso político: intelectuales de izquierda en la Argentina, 1918-1955, Bernal, Editorial Universidad Nacional de Quilmes, 2008, 41-42. Las cifras informadas se obtienen de Pablo Buchbinder, Historia de las universidades argentinas, Buenos Aires, Sudamericana, 2010, 118; y Moyano, op. cit., 67.

13 Para apreciar cómo se verificaban las tensiones entre teocentrismo-antropocentrismo y escolásticaempirismo se sugiere revisar el dossier de Políticas de la memoria dedicado a las conferencias que realizara Saúl Taborda en junio de 1918 y, muy especialmente, la interpretación que ahí presenta Fernando Diego Rodríguez, Un domingo en la vida de Saúl Taborda, Políticas de la memoria, $\mathrm{n}^{\circ} 16$, Buenos Aires, verano 2015/2016, 271. Quien desee comprender en profundidad como se escenificaban las diferentes perspectivas científicas e ideológicas al interior de las universidades argentinas, entre ellas especialmente las pugnas suscitadas en la Universidad de La Plata, revisar el trabajo de Gustavo Vallejo, Escenarios de la cultura científica en Argentina: ciudad y universidad (1882-1955), Madrid, Consejo Superior de Investigaciones Científicas, 2007. 
significa que es desde estas décadas que una parte significativa del estudiantado universitario comienza a asumir que tiene tanto que decir sobre la sociedad, sus problemas y las formas de encararlos, como toda persona dedicada a los oficios del pensamiento. Una concepción que se fortalecerá con el paso de los años gracias a los mecanismos de socialización propios del campo intelectual, entre los cuales tuvieron un lugar destacado las publicaciones especializadas y los congresos entre pares ${ }^{14}$.

\section{Fases e hitos del movimiento}

Identificadas algunas de las principales coordenadas históricas que enmarcaron al movimiento cordobés están dadas las condiciones para proceder a una caracterización general del mismo. Tarea que se realizará distinguiendo tres fases dentro del movimiento, las que difieren, en lo substancial, en la expansión o alcance que éste adquiriría ${ }^{15}$.

En la primera fase, que se prolongó desde noviembre de 1917 hasta los primeros días de abril de 1918, el conflicto pasó de ser un asunto puntual, defensivo y/o reactivo, es decir, que solo afectaba a una parte del estudiantado, a otro en que la totalidad de la comunidad universitaria se vio involucrada.

Todo comenzó cuando al finalizar el año escolar de 1917 los estudiantes de medicina e ingeniería emprendieron protestas por asuntos puntuales. Los de ingeniería exigían que se revocaran las penalidades impuestas a quienes tuvieran baja asistencia o no aprobaran las materias y los de medicina demandaban que se diera pie atrás a la resolución de cerrar el Internado del Hospital de Clínicas ${ }^{16}$. Y como suele ocurrir en este tipo de

\footnotetext{
${ }^{14}$ Martín Bergel, Latinoamérica desde abajo: las redes transnacionales de la Reforma Universitaria, Emir Sader y otros (comps.) La Reforma Universitaria: desafios y perspectivas noventa años después, Buenos Aires, Consejo Latinoamericano de Ciencias Sociales, 2008, 154-156. Entre los encuentros que tuvieron directa relación con los sucesos estudiados se cuentan las tres versiones de los Congresos de Estudiantes Americanos - Montevideo 1908, Buenos Aires 1910 y Lima 1912-, el Primer Encuentro Nacional de Estudiantes Universitarios realizado en Córdoba en 1918 y el Primer Congreso Internacional de Estudiantes efectuado en Ciudad de México en 1921. Entre los congresos que se vincularon indirectamente al tema estudiado se cuentan el Primer Congreso Nacional de Estudiantes realizado en Ciudad de México en 1910, el Congreso Nacional de Estudiantes efectuado en Perú en 1921 y el Primer Congreso Nacional de Estudiantes realizado en Cuba en 1923. Es posible encontrar referencias de encuentros estudiantiles universitarios desde 1908 hasta 1995 en Ricardo Romero, El movimiento estudiantil argentino en el siglo XX, Buenos Aires, EUDEBA, 1998, 125 y siguientes.

${ }^{15}$ Los hechos que sustentan la periodificación expuesta, de elaboración propia, fueron tomados de algunas de las crónicas mejor documentadas sobre los sucesos de Córdoba, entre ellas, Pablo Buchbinder, ¿Revolución en los claustros? La Reforma Universitaria de 1918, Buenos Aires, Sudamericana, 2008, 87-115; Buchbinder, op. cit., 2010, 92 y siguientes; Caldelari y Funes, op. cit., 15-19, Alberto Ciria y Horacio Sanguinetti, La Reforma Universitaria (1918-2006), Santa Fe, Universidad Nacional del Litoral, 2006, 28-41; Ferrero, op. cit., 15-22; Jorge Las Heras, El grito de Córdoba: la Reforma Universitaria de 1918 y su vigencia en la universidad del siglo XXI, Santiago de Chile, Sudamericana, 2009, 19-42; Romero, op. cit., 1998; y Tünnermann, op. cit., 65-68.

${ }^{16}$ La exigencia relativa al internado no solo informa de la existencia de un problema educacional -en la medida que reaccionaba ante la clausura de un espacio de formación para los futuros profesionales-, también da cuenta de la pérdida de beneficios económicos para esos estudiantes que venían de puntos distantes y tenían dificultades para solventar sus gastos de residencia.
} 
movimientos, los hechos que los provocan, sus detonantes, fueron subestimados por las autoridades universitarias.

En marzo de 1918, luego de retornar del receso estival, el estudiantado conformó una agrupación, el Comité Pro Reforma Universitaria, liderada por representantes de las tres facultades existentes: ingeniería, medicina y derecho. Organización que hizo un eficaz llamado a boicotear las actividades regulares de la universidad mediante un paro general y la no matriculación. Tanta fuerza tuvieron estas medidas de presión que a comienzos de abril las autoridades universitarias decidieron clausurar la institución con estos argumentos:

Atento [a] los reiterados actos de indisciplina que públicamente vienen realizando los estudiantes de las distintas facultades de la Universidad, como ser: inasistencia colectiva a las clases, medios violentos para impedir la matriculación de alumnos, falta de respeto a la persona de académicos y profesores, manifestaciones notorias de rebeldía contra las autoridades del instituto, todo lo cual ha perturbado la función docente (...) resuelve: clausurar las aulas de la Universidad Nacional de Córdoba hasta nueva resolución del H. Consejo Superior. Queda prohibida la entrada al instituto a toda persona que no sea académico, profesor o empleado del mismo ${ }^{17}$.

Así, en tan solo unos meses el descontento estudiantil se había transformado en movimiento y éste había dejado al descubierto la incapacidad de las autoridades universitarias para resolver el conflicto. A comienzos de abril de 1918, por tanto, la crisis en la Universidad de Córdoba ya estaba declarada.

En la segunda fase del movimiento, que va desde comienzos de abril hasta mediados de junio de 1918, las movilizaciones van a alcanzar una dimensión nacional. Condición que adquiere, fundamentalmente, gracias a la buena acogida que tuvieron los llamados que hiciera el estudiantado cordobés para que los universitarios y las autoridades del país tomaran una posición activa frente a sus demandas. Entre las muestras más consistentes de solidaridad que el estudiantado argentino manifestó hacia sus pares cordobeses se cuenta la fundación, el 11 de abril de ese año, de la primera organización estudiantil universitaria de alcance nacional, la Federación Universitaria Argentina ${ }^{18}$. El poder ejecutivo, en tanto, también resolvió tomar cartas en el asunto y ese mismo 11 de abril anunciaba su decisión de intervenir la universidad. El interventor, luego de apersonarse en Córdoba para interiorizarse de las demandas estudiantiles — las cuales, para

\footnotetext{
${ }^{17}$ En Ciria y Sanguinetti, op. cit., 28.

18 Se debe advertir que las organizaciones estudiantiles con fines mutuales, gremiales y/o culturales proliferaron en América Latina desde fines del siglo XIX. Éstas, al ir creciendo en número de asociados se fueron complejizando y desarrollaron un accionar coordinado con otras entidades similares. Proceso de aglomeración del cual van a surgir varias organizaciones nacionales como la indicada e, inclusive, algunas de carácter internacional. En Córdoba, en particular, desde 1901 hay antecedentes de asociaciones estudiantiles con miembros de las tres facultades existentes — medicina, derecho e ingeniería - . En la capital federal, por su parte, desde 1908 funciona la Federación de Estudiantes de Buenos Aires. En el plano internacional entre 1908 y 1912 se registran esfuerzos sistemáticos por implementar una Liga de Estudiantes Americanos y ya en 1921 se conforma en México una entidad aún más ambiciosa, la Federación Internacional de Estudiantes.
} 
ese entonces, ya habían superado a las exigencias iniciales e incluían, entre otros puntos, la modificación del gobierno universitario-, decretó la reorganización de la universidad conforme a los lineamientos que desde 1906 operaban en la Universidad de Buenos Aires. Directrices que, en lo fundamental, hacían que el poder en la universidad dejara de ser monopolio de un reducido grupo de consejeros que no tenían, necesariamente, un vínculo orgánico con la institución, para pasar ahora a manos del profesorado.

El 15 de junio, cuando los docentes debían elegir a su máxima autoridad conforme a los nuevos estatutos, el estudiantado interrumpió intempestivamente la votación. Una vez más los estudiantes declararon la huelga general, esta vez por juzgar que de concluirse la elección los mismos de siempre terminarían dirigiendo a la universidad. Esta fecha, desde entonces, es recordada como el día de "la reforma universitaria".

En la tercera fase del movimiento, la cual se prolongó desde mediados de junio hasta comienzos de octubre de 1918, éste ampliará su radio de acción e interpelará a diferentes actores sociales. De hecho, fue a partir de la malograda elección del rector que las protestas adquirieron nuevos bríos y lograron concitar la solidaridad de algunos sindicatos obreros de la ciudad. Esta fase tiene entre sus principales hitos la aparición, el 21 de junio, de una declaración pública que condensa parte importante del ideario que motivara a la juventud movilizada. Declaración que fue suscrita por la Federación Universitaria de Córdoba, novel organización que desde el 16 de mayo reemplazaba al Comité Pro Reforma Universitaria, y que es conocida como el "Manifiesto Liminar". Documento que apareció en una edición extraordinaria de La Gaceta Universitaria -órgano de difusión del estudiantado cordobés-, también en el diario local La Voz del Interior, y que, como señala el historiador Martín Bergel, obtuvo resonancia en numerosos medios de toda América Latina ${ }^{19}$.

Otro acontecimiento sustantivo de esta fase fue la realización, entre los días 20 y 31 de julio, del Primer Congreso Nacional de Estudiantes Universitarios. Instancia convocada por la Federación Universitaria Argentina y que congregó en Córdoba a representantes de las cinco universidades del país (Buenos Aires, Córdoba, Litoral, La Plata y Tucumán). El documento donde se plasmaron las conclusiones de dicho encuentro, mirado retrospectivamente, condensa las principales demandas cordobesas, argentinas y latinoamericanas de entonces: participación estudiantil en el gobierno universitario, reestructuración del proceso de selección docente y generación de vínculos más estrechos entre la universidad con su entorno social. Demandas que se estudiarán en profundidad en el próximo apartado.

El último de los acontecimientos decisivos de esta fase, y por lo tanto del movimiento, se efectuó el 9 de septiembre. Ese lunes el estudiantado se tomó la rectoría de la universidad, nombró autoridades provisorias entre los mismos estudiantes e invitó a los vecinos de la ciudad a participar de la reapertura de la institución. Con otras palabras, se asumió como agente capaz de liderar procesos sistemáticos de enseñanza-aprendizaje. A poco andar la toma terminó abruptamente cuando un contingente de policías y militares detuvo a todos los manifestantes. Pese a este desenlace, la medida de presión cumplió con su cometido pues el gobierno decidió intervenir nuevamente la universidad y designó para

\footnotetext{
${ }^{19}$ Bergel, op. cit., 165. 
tal efecto al mismísimo Ministro de Justicia e Instrucción Pública. Intervención que atendió parte importante de las demandas estudiantiles, sobre todo las relativas a su participación en el gobierno universitario, y que, por lo mismo, se entiende como el epílogo del movimiento.

Cabe recordar, además, que en el resto del país en estos años también se estaban sucediendo movimientos estudiantiles significativos, prueba de ello es que pocas semanas antes del desenlace cordobés los estudiantes bonaerenses conseguían reformas en línea con las descritas, que en 1919 el estudiantado platense intensificará sus movilizaciones motivado por el sonado triunfo en el interior del país y que en la década de los veinte los santafecinos y tucumanos no solo lograrán nacionalizar sus universidades, hasta entonces provinciales, también conseguirán participación en el gobierno universitario. A nivel latinoamericano, en tanto, se apunta que en 1919 los estudiantes limeños, reconociéndose herederos de sus pares cordobeses, iniciaron una movilización que se prolongó por varios años y que se convertirá en otro de los capítulos más significativos de las luchas estudiantiles del continente. Y se apunta, a su vez, que luego de los sucesos peruanos hubo variados movimientos en Bolivia, Chile, Cuba, México, Paraguay y otros países de la región alentados, entre otros factores, por los postulados popularizados en Córdoba ${ }^{20}$.

\section{Las principales demandas del movimiento cordobés}

Como se adelantara, el documento donde se sistematizan las discusiones de aquel congreso estudiantil realizado en medio del movimiento de Córdoba permite identificar tres tipos de demandas: modificar el gobierno universitario, reestructurar los procedimientos de conformación del profesorado y aproximar la universidad a su entorno social. Sobre cada una de ellas se entregarán más antecedentes.

\section{a) Modificación del gobierno universitario}

Para comprender la exigencia de modificar el gobierno universitario es necesario remontarse al menos hasta 1885, fecha en que se promulgó la ley que regía a las universidades nacionales ${ }^{21}$ y que prescribía, en lo fundamental, que las decisiones sobre los asuntos más sensibles de la institución, los relativos a docencia, planes de estudio, exámenes, entre otros, descansarían en consejos — llamados en ese tiempo academiascompuestos por personas de alta figuración pública que ejercerían dicha función de por vida.

Casi desde su misma promulgación el estudiantado fue elaborando un diagnóstico crítico sobre las disposiciones de esta ley, el cual estaba ya plenamente maduro en 1918. Se evaluaba que esta forma de gobierno universitario era tan ineficiente que llegaba a ser

\footnotetext{
${ }^{20}$ Véanse Renate Marsiske, Movimientos estudiantiles en América Latina: Argentina, Perú, Cuba y México, 1918-1929, Ciudad de México, CESU/UNAM, 1989, 35 y siguientes; y Juan Carlos Portantiero, op. cit., 58 y siguientes.

${ }^{21}$ Véase Adriana Puiggrós, ¿Qué pasó en la educación argentina? Breve historia desde la conquista hasta el presente, Buenos Aires, Galerna, 2003, 88 ; y Tedesco, op. cit., 97-98.
} 
ineficaz, en la medida que los consejeros no conocían a fondo el quehacer universitario, no velaban por los intereses de la institución -sino más bien por la conveniencia de los distintos grupos de poder provincial/nacional a los que adscribían- y no consideraban los aportes que podía realizar una parte central de la comunidad educativa, el estudiantado ${ }^{22}$. Todo lo cual incidió en que se juzgara que la institucionalidad universitaria no estaba acompañando los cambios en el país, ni estaba a la altura de los retos de una universidad que crecía decididamente.

Todas las fórmulas que el estudiantado barajó para acabar con esta "oligarquía universitaria" incluyeron la conformación de un entramado de poder compuesto por el conjunto de actores interesados en el porvenir de la institución: estudiantes, docentes y egresados/as. Donde lo novedoso era, ciertamente, la inclusión de representantes estudiantiles. Aunque se comparten las interpretaciones que invitan a pensar que dicha demanda habría sido resultado del desengaño que significó para los estudiantes la actuación de los profesores en la malograda elección del rector aquel 15 de junio de 1918 -tesis popularizada, entre otros, por quien fuera en ese entonces dirigente estudiantil platense, Julio V. González ${ }^{23}$-, es necesario recalcar que la defensa del co-gobierno estaba presente entre los estudiantes latinoamericanos al menos desde diez años antes. De hecho, entre todos los antecedentes que respaldan esta aseveración el más decisivo fue la discusión que se dio en el marco del Primer Congreso de Estudiantes Americanos efectuado en 1908 en Montevideo. Pues ahí quedó en evidencia, por un lado, que la incorporación de estudiantes al gobierno universitario estaba siendo debatida simultáneamente en Argentina, Brasil, Perú y Uruguay y, por otro lado, que éste era un asunto que concitaba amplios grados de consenso entre los más de cien representantes estudiantiles que participaron de dicho congreso $^{24}$.

En la Universidad de Córdoba, como en otras casas de estudio de la región, para viabilizar la inclusión de los estudiantes en el gobierno institucional se exploraron diversas modalidades. Modalidades que diferían, principalmente, en la proporción de representantes estudiantiles que tendrían los órganos colegiados, en los requisitos que éstos debían satisfacer y en las atribuciones que gozarían -es decir, si contarían con derecho a voz y a voto, o solamente a voz-. Una de las variantes que más trascendería fue la que se consolidó precisamente en el congreso de julio de 1918 en Córdoba: conformar instancias donde el poder se distribuyera en partes iguales entre estudiantes, egresados/as y docentes. El pasaje

\footnotetext{
${ }^{22}$ Gabriel Del Mazo, Estudiantes y gobierno universitario: bases doctrinarias y técnica representativa en las universidades argentinas y americanas, Buenos Aires, Librería y Editorial "El Ateneo", 1946, 10.

${ }^{23}$ González, op. cit., 191.

24 Véase Vania Markarian, María Eugenia Jung \& Isabel Wschebor, 1908 el año augural, Montevideo, Archivo General de la Universidad de La República, 2008a, 97-136. Otros indicios que denotan lo extendida que era esta preocupación son los que refieren a que desde 1908 había representación estudiantil en el gobierno universitario uruguayo, que desde 1910 existía vocería estudiantil en las instancias decisivas de la Universidad Nacional de México, que en 1913 una comisión de estudiantes cordobeses discutió esta materia en el marco de los festejos del tercer centenario de la universidad, que en 1916 la Federación de Estudiantes de Buenos Aires solicitó formalmente la inclusión de representantes en los órganos de decisión universitaria y que en 1917 el diputado nacional argentino Jorge Raúl Rodríguez presentó un proyecto de ley donde se contemplaba este tipo de participación, Del Mazo, op. cit., 1946, 27-29.
} 
que se transcribe a continuación, tomado del informe de la comisión que redactó las conclusiones de dicho congreso, ayuda a comprender los razonamientos que sustentaban esta lógica de los tres tercios:

Para evitar la formación de círculos y excluir toda preponderancia, cree la comisión que sólo puede adoptarse un sistema y es el de evitar en los cuerpos directivos el predominio de cualquiera de los 'estados' universitarios. Hemos convenido en llamar 'estados' a los diferentes cuerpos de estudiantes, profesionales y profesores, por cuanto la soberanía universitaria reside en el conjunto de estos diversos núcleos, cuyos derechos no son proporcionales al número de sus componentes. El sufragio, medio por el cual intervienen periódicamente en el gobierno de la universidad, no es proporcional al número de sufragantes, siendo la representación fija e invariable. Se establece por tal medio, no la democracia del número, sino la legítima y proporcional representación de los intereses. La elección directa por todos los miembros de la facultad dejaría, por otra parte, en minoría a los del cuerpo de profesores; y aunque ellos podrán ser los elementos menos capaces para la fiscalización que en primer término debe ser, como se ha dicho, de sí mismos, no deben ser excluidos, a juicio de la comisión, porque su propia posición supone que poseen valiosas cualidades científicas y de experiencia.

Por dichas razones la comisión cree que es garantía necesaria para el progreso de las universidades, la formación de los consejos directivos por igual número de representantes de los diplomados, los estudiantes y los profesores que impedirá la formación de círculos preponderantes a la par que obligará a una acción solidaria y concurrente de elementos que hoy parecen antagónicos ${ }^{25}$.

\section{b) Reestructuración de los procedimientos para conformar al profesorado}

La exigencia de reformular los mecanismos para formar parte del profesorado se apoyaba, fundamentalmente, en la comprensión de que los méritos no estaban siendo un criterio sustantivo en la conformación del cuerpo docente de la universidad. Situación que incidía en que muchas veces los profesores postergaran sus compromisos con la institución en favor de sus actividades profesionales -lo que se traducía en que no impartieran sus clases o bien no las prepararan adecuadamente- ${ }^{26}$. Este irregular ejercicio de la docencia influía en que se perpetuaran estrategias pedagógicas que no exigían mayor compromiso por parte del maestro, como la repetición, y que no redituaban aprendizajes significativos para el

\footnotetext{
${ }^{25}$ En Del Mazo, op. cit., 1967, 55.

${ }^{26}$ Incluso en casos extremos, como lo denunciara el estudiantado cubano de la década de 1920, los profesores comprometían su honra al vender las calificaciones o al exigir a sus estudiantes que asistieran a sus clases particulares/pagadas. Al respecto véase, por ejemplo, Yasmín Cuevas y Guadalupe Olivier, Julio Antonio Mella: de líder universitario a activista social, Renate Marsiske (coord.) Movimientos estudiantiles en la historia de América Latina, volumen III, Ciudad de México, CESU/UNAM/Plaza y Valdés Editores, 2006, 108-109.
} 
estudiante, caso de la memorización. Escenario que en innumerables ocasiones llevó al estudiantado a sentenciar que la universidad solo estaba siendo una "fábrica de profesionales" donde únicamente se aprendía a velar por los intereses propios. Por lo tanto, cuando en el Manifiesto Liminar se clamaba por acabar con esa universidad que servía de refugio a docentes mediocres e ignorantes, también se expresaba la voluntad de contar con profesores que defendieran perspectivas hasta entonces marginadas del quehacer universitario, entre ellas las que ponían su atención en la comprensión y solución de las problemáticas sociales. ¿Cómo lograrlo? El movimiento cordobés, como muchos de los que le secundaron, propuso dos innovaciones íntimamente relacionadas: la libre docencia y la asistencia voluntaria a clases.

Instaurar la libre docencia aludía a la creación de mecanismos que permitieran que cualquier persona que deseara impartir un curso universitario, y que tuviera las competencias para ello, pudiera hacerlo. La libre docencia también fue vista, como sugiere el historiador Pablo Buchbinder, como el primer eslabón de una incipiente "carrera docente", en el sentido que debía constituirse en el canal regular para acceder a la docencia universitaria remunerada ${ }^{27}$. Esta libre docencia se constituía, por tanto, en una manera de transparentar el proceso de selección del profesorado, de renovar al cuerpo docente y de estimular la sana competencia entre los catedráticos - pues ahora deberían esforzarse por atraer a ese estudiante que tendría más cursos donde escoger-. Y para que el estudiante pudiera efectivamente elegir entre los cursos impartidos por docentes con y sin remuneración, se entendía que éstos debían tener un mismo estatus formal y que no se debía exigir asistencia, es decir, que no podía ser obligatoria ni la inscripción en los cursos ni la participación en los mismos. En una comunicación que el estudiantado cordobés dirigió al Consejo Superior de su universidad el 1 de abril de 1918 refieren ilustrativamente a esta simbiosis entre libre docencia y asistencia voluntaria:

Es indudable y nos adelantamos a concederlo que - con la actual organización disciplinaria - es de todo punto imposible que pueda progresar la libre docencia. En efecto, bajo el imperio de la asistencia obligatoria sistema infantil y colegialesco - el alumno está imposibilitado para hacer acto de presencia en los cursos libres, aun cuando el profesor que los dicte le ofrezca las mayores garantías de honorabilidad y competencia, desde que implica una sobrecarga la duplicidad de los cursos. Por el contrario, si se adopta la asistencia libre, el alumno interesado en oír a los mejores, no vacilará en optar por ellos y se producirá, a no dudarlo, una saludable selección. El mal profesor, aun cuando se encuentre escudado en un nombramiento oficial, tendrá que eliminarse al contemplar el aula desierta, y

\footnotetext{
${ }^{27}$ Interpretación obtenida de Buchbinder, op. cit., 2008, 156; y Buchbinder, op. cit., 2010, 109. A veces el sentido común puede llevar a confundir docencia libre, cursos libres y libertad de cátedra. Docencia libre remite a esta posibilidad de que quien este apto/a para impartir cursos en la universidad pueda hacerlo en caso de que lo desee; cursos libres hace alusión a aquellos que no están destinados únicamente a estudiantes sino más bien a cualquier interesado/a; y libertad de cátedra refiere al derecho que tendrían los docentes de no ser censurados en lo que a contenidos, métodos y uso de recursos pedagógicos se refiere.
} 
conocer por este medio la tácita pero elocuente expresión del concepto que merece a sus alumnos ${ }^{28}$.

Otra de las argumentaciones con que el estudiantado defendió la necesidad de la asistencia libre refería a que así se permitiría que quienes estudiaran y trabajaran —una combinación que era, todo lo indica así, cada vez más frecuente — , pudieran desempeñar apropiadamente ambas funciones. Criterio que da luces también de los cambios en la composición social que estaba viviendo el estudiantado y también, aunque indirectamente, de la emergencia de esta nueva sensibilidad social presente entre los estudiantes ${ }^{29}$.

\section{c) Aproximación de la universidad a su entorno social}

La demanda por acercar la universidad a la sociedad se apoyaba en comprensiones que veían que la institución se estaba convirtiendo en una torre de marfil y no, como aspiraban sectores cada vez más significativos del mundo estudiantil, en un polo cultural que irradiara conocimientos que favorecieran la solución de los múltiples problemas que afectaban a parte importante de la población. Una preocupación inscrita en los mismos horizontes que desde fines del siglo XIX poseían las experiencias que se proponían educar a los sectores populares, pues por un lado se procuraba que la universidad contribuyera a iluminar, civilizar o modernizar al "salvaje", "ignorante" y/o "amoral" y, por otro lado, se aspiraba a que ella ayudara a empoderar, fortalecer o emancipar al "oprimido", "subyugado" y/o "explotado". Si bien tanto ilustrar como emancipar podían leerse como "liberar de las cadenas de la ignorancia", debido a que desde las últimas décadas del siglo XIX proliferaban discursos demócratas, socialistas, anarquistas y comunistas —amplificados aún más con el triunfo de la Revolución Rusa en 1917-, con el correr de los años estos horizontes fueron profundizando sus particularidades o, si se quiere, acentuando sus diferencias. Cuestión que fue haciendo que cada vez más "ilustrar" fuera enseñar a los sectores populares las claves para desenvolverse en la sociedad, mientras que "emancipar" fuera entregarles herramientas para que puedan transformarla. Así, aunque todas las iniciativas de educación popular buscaran ilustrar y emancipar simultáneamente, lo que las diferenciaba era el énfasis dado a cada una de estas orientaciones.

En el ambiente universitario de principios del siglo $\mathrm{XX}$, las experiencias de educación popular que privilegiaban un acercamiento ilustrado se conocieron como "extensión universitaria" y las que enfatizaban la dimensión emancipadora se identificaron como "universidades populares". En general las experiencias de extensión universitaria fueron asumidas como política institucional por las universidades, mientras que las universidades populares fueron impulsadas por organizaciones estudiantiles (o intelectuales) que no tenían necesariamente vínculos formales con una institución de educación superior. Tendencias que, dicho sea de paso, perviven hasta la actualidad.

\footnotetext{
${ }^{28}$ En Del Mazo, op. cit., 1967, 18.

${ }^{29}$ Comprensión presente, entre otras obras, en Portantiero, op. cit., 90.
} 
En el congreso estudiantil de Córdoba en 1918 ambas vías de acercamiento a los sectores populares fueron demandadas. Ahí se planteó la necesidad de que la extensión universitaria se implementara de manera oficial por las universidades, proponiéndose la creación de un "Comité Social Universitario" que coordinara el acercamiento a la comunidad mediante conferencias, visitas a museos, presentaciones artísticas, entre otras. Y se planteó también la conveniencia de que los estudiantes crearan una "Oficina de Acción Social" que articulara las iniciativas dirigidas hacia la comunidad, tales como cursos, conferencias, publicaciones, etcétera ${ }^{30}$. En las conclusiones que se aprobaron en dicho congreso, publicadas con fecha 12 de agosto de 1918 en La Gaceta Universitaria, estos puntos se expresaron de la siguiente forma:

"El P.C.N de E.U [Primer Congreso Nacional de Estudiantes Universitarios] recomienda a las universidades del país y a las distintas federaciones estudiantiles, se avoquen al estudio, conjuntamente, a objeto de implantar la extensión universitaria, con carácter oficial [...]. Recomienda, asimismo, a todos los egresados y alumnos de la universidad que actúen en centros no universitarios, la organización de cursos de extensión universitaria [...] Resuelve: $1^{\circ}$. Encomendar a los centros estudiantiles representados, la iniciación de una campa [sic] eficaz en contra del analfabetismo, organizando colegios nocturnos para obreros, con la colaboración exclusiva de estudiantes y en los que se impartirán las enseñanzas correspondientes a los primeros grados de las escuelas comunes. $2^{\circ}$. Encargar a las federaciones universitarias soliciten de las autoridades de sus respectivas universidades, se haga efectiva la extensión universitaria, organizando al efecto en cada una de las facultades series de conferencias dominicales sobre temas de interés práctico para el pueblo y a desarrollarse preferentemente en locales obreros. $3^{\circ}$ Declarar la necesidad de que los universitarios presten su colaboración de una campaña en favor de la higiene social en todo el país. A tal efecto encomienda a las federaciones adheridas, organicen una 'oficina de acción social' [...] que tendrá la misión de organizar a los universitarios que quieran colaborar en esa acción, divulgando por medio de conferencias, el folleto, el periodismo, principios de higiene particular y pública, profilaxis de enfermedades infecciosas, efectos de vicios públicos, etc."31

Con miras a obtener una imagen un poco más completa sobre esta demanda por acercar la universidad a la sociedad se reseñarán, brevemente, dos de las experiencias mejor documentadas de educación popular impulsadas por universitarios latinoamericanos en estos años, una de tinte ilustrada, otra de cariz emancipador.

La Universidad Popular Mexicana, fundada en 1912, fue implementada por jóvenes intelectuales que entendían que las tareas de extensión universitaria no podían emprenderse desde la Universidad Nacional debido, fundamentalmente, a que ésta se encontraba

\footnotetext{
${ }^{30}$ Del Mazo, op. cit., 1967, 67-68.

${ }^{31}$ La Gaceta Universitaria, ${ }^{\circ} 12,1918$, pp. 2.
} 
absorbida en la tarea de no naufragar en el convulsionado contexto revolucionario. Por esto, pese a su denominación, se le entiende más como una experiencia de "extensión universitaria" que como una "universidad popular". En sus mejores momentos esta institución impartió clases vespertinas todos los días y contó con una asistencia media de noventa personas. Además de los cursos sistemáticos, los que duraban entre cinco y siete sesiones, se dictaban conferencias a cargo de connotadas personalidades, se facilitaban libros a través de su biblioteca y se generaban materiales de estudio en su imprenta/editorial. Entre las materias que se impartieron destacan las de orientación práctica como electricidad, higiene o taquigrafía, y las de signo humanista como las bellas artes, la historia o la sociología. El fin de esta iniciativa, en 1920, coincide con la llegada de José Vasconcelos a la rectoría de la Universidad Nacional, pues al fortalecer la extensión universitaria en la institución tornó dispensables los afanes de su par popular ${ }^{32}$.

La Universidad Popular Manuel González Prada, en tanto, fue fundada en enero de 1921 por la Federación de Estudiantes del Perú -organización activa desde 1916- y en pocos años contó con locales en más de cinco ciudades ${ }^{33}$. Entre sus profesores figuraban estudiantes de la Universidad Mayor de San Marcos, como Víctor Raúl Haya de la Torre, quien fuera presidente de la Federación de Estudiantes del Perú, y otros colaboradores ajenos al mundo universitario como José Carlos Mariátegui, uno de los fundadores del Partido Socialista Peruano. En sus primeros años, los que a la postre resultaron ser los más activos, se registraron masivas concurrencias de obreros a sus actividades -entre las que destacaban los cursos, conferencias, fiestas y excursiones-. Aunque esta universidad también tuvo un afán iluminista, no por nada algunas de las materias impartidas pertenecían al mundo de las ciencias o las artes, su principal ambición fue la emancipación. Cuestión que se refleja en algunos de los contenidos que se trataban -como por ejemplo los referidos a organización obrera-, en el nombre de la propia universidad -que homenajeaba a uno de los intelectuales peruanos más combativos de esas décadas- y en su lema "La Universidad Popular no tiene más dogma que la justicia social”.

Cabe consignar que en América Latina las universidades populares han tenido presencia desde principios del siglo XX y que en 1917 también hubo una experiencia de esta naturaleza en Córdoba. Universidad popular que, pese a su breve existencia, pudo impartir cursos y conferencias sobre higiene, moral o economía política en locales obreros $\mathrm{y}$ en salones facilitados por escuelas primarias ${ }^{34}$.

\footnotetext{
${ }^{32}$ Morelos Torres Aguilar, Cultura y revolución: la Universidad Popular Mexicana (Ciudad de México, 1912-1920), Ciudad de México, Universidad Nacional Autónoma de México, 2009, 32 y siguientes.

33 Informaciones extraídas, principalmente, Jeffrey Klaiber, The popular universities and the origins of Aprismo, 1921-1924, The Hispanic American Historical Review, Vol. 55 (4), Durham, 1975, 693-715; Enrique Cornejo, Crónica del movimiento estudiantil peruano, Juan Carlos Portantiero, Estudiantes y política en América Latina: el proceso de la Reforma Universitaria (1918-1930), Ciudad de México, Siglo XXI Editores, 1987 [1926], 232-266; Víctor Raúl Haya de la Torre, La Reforma Universitaria, Dardo Cúneo (comp.) La Reforma Universitaria (1918-1930), Caracas, Biblioteca Ayacucho, 1988 [1929], 232-240; Mariátegui, op. cit.; Ricardo Melgar, Las universidades populares en América Latina, 1910-1925, Estudios, Vol $^{\circ}$ 11-12, Córdoba, 1999, 41-57, entre otros.

${ }^{34}$ Véase María Alejandra Navarro, Los jóvenes de la ‘¡Córdoba Libre!’: un proyecto de regeneración moral y cultural, Ciudad de México, Nostromo, 2009, 125; y Ferrero, op. cit., 14.
} 


\section{La dimensión social del movimiento: pistas complementarias}

Aunque fueron las demandas por fortalecer la extensión universitaria y crear universidades populares las muestras más contundentes de la nueva sensibilidad social que tendrá el estudiantado, hay otras pistas que permiten dimensionar mejor los matices que adquirió este espíritu de época: el trasfondo de algunas discusiones que se dieron al interior del movimiento y los acercamientos concretos de los estudiantes movilizados a los sectores populares.

Sobre los debates surgidos al interior del movimiento se precisa que, más allá de las diferencias que existían en aspectos sensibles como lo oportuna que sería la movilización o cuáles debían ser los objetivos perseguidos, ellos también dejan entrever una preocupación por acercar la universidad a la sociedad ${ }^{35}$. Por ejemplo, entre quienes estaban a favor de las movilizaciones existían dos posiciones, los que como Osvaldo Laudet, presidente de la recién creada Federación Universitaria Argentina, pensaban que el accionar estudiantil debía enmarcarse en los asuntos universitarios por comprender que éstos eran neutrales, técnicos o apolíticos. Una posición que queda bien reflejada en las palabras que dio en el Primer Congreso Nacional de Estudiantes Universitarios: "Señores: éste es un congreso universitario y ha de estudiar los problemas con espíritu universitario. Quiero decir, que todo es ajeno a él, menos las cuestiones de pedagogía superior y que todas ellas han de ser resueltas con espíritu científico"36. Y estaban también los que entendían que los problemas universitarios no se podían resolver sin antes haber transformado a la sociedad. Una posición que tuvo muchos exponentes y, en consecuencia, presentó una infinidad de matices $^{37}$. Conforme recuerda la historiografía latinoamericana uno de los promotores más vehementes de ésta última posición fue el dirigente estudiantil cubano Julio Antonio Mella, quien sostenía, por ejemplo, que era "necesario primero una revolución social para hacer una revolución universitaria"38.

\footnotetext{
${ }^{35}$ Véase Natalia Bustelo y Lucas Domínguez Rubio, Vitalismo libertario y Reforma Universitaria en el joven Carlos Astrada, Políticas de la memoria, n 16, Buenos Aires, verano 2015/2016, 297-298.

${ }^{36}$ Osvaldo Laudet, Discurso del Presidente de la Federación Universitaria Argentina Osvaldo Laudet en el I Congreso Nacional de Estudiantes, Ricardo Romero (ed.) El movimiento estudiantil argentino en el siglo XX, Buenos Aires, EUDEBA, 1998 [1918], 89.

37 Algunas aproximaciones, aunque referidas principalmente al caso del estudiantado platense, pueden encontrarse en la obra de Graciano, op. Cit., 56, 58, 59 y 66. Se apunta, a su vez, que Juan Carlos Portantiero, con frases como el movimiento estudiantil "o es un capítulo de la reforma social o termina triturada por los intereses de los poderososo", es uno de los exponentes analíticos más consistentes de esta posición. Véase Portantiero, op. cit., 62.

38 Julio Antonio Mella, ¿Puede ser un hecho la Reforma Universitaria? Emir Sader y otros (comps.) La Reforma Universitaria: desafios y perspectivas noventa años después, Buenos Aires, Consejo Latinoamericano de Ciencias Sociales, 2008 [1925], 217. Estas perspectivas se han mantenido vigentes hasta la actualidad y, en algunos momentos, incluso han gozado de posiciones hegemónicas. En el Segundo Congreso Nacional de Estudiantes Universitarios, realizado en Buenos Aires en 1932, es posible apreciar cómo el estudiantado argentino va transitando desde la primera a la segunda postura, pues ahí se concluye que las transformaciones universitarias debían comprenderse como parte indivisible de los cambios en la sociedad. Quienes deseen acercarse en profundidad al devenir entre reforma y revolución dentro del
} 
Otro debate donde esta nueva sensibilidad hacia las problemáticas sociales mostró también presencia fue en el relativo al financiamiento universitario. Sí, porque en el encuentro de Córdoba en 1918 había quienes proponían, por un lado, que la totalidad del financiamiento universitario proviniera de las arcas fiscales — sugiriéndose inclusive mecanismos para recaudar dichos recursos - y, por otro lado, que las organizaciones estudiantiles coordinaran dispositivos de recolección de fondos entre privados para costear becas a sus miembros con menores recursos económicos. El sustrato social de estos postulados se puede apreciar más claramente en una de las intervenciones que realizaron los promotores de ésta posición:

La universidad debe ser amplia y abierta. Con el primer término, "amplia", quiero significar que ella debe ser tan comprensiva como el país mismo, porque los verdaderos laboratorios de la universidad argentina deben ser el territorio entero de la nación argentina y de la nación americana en toda su extensión, en toda la realidad palpitante del hombre y de la naturaleza. Con el segundo término "abierta" quiero expresar mi idea de que la universidad debe abrirse a todas las aptitudes y a todas las vocaciones, sin malograr la de los pobres, dificultándose la entrada, sino por el contrario, estableciendo la gratuidad para no agravarles la desigualdad existente en otro orden de cosas, que ya es de por sí suficientemente injusto. Sostengo que una de las formas más antipáticas e irritantes del privilegio universitario lo constituye ese hecho, señor presidente, de que los jóvenes menos favorecidos por la fortuna, no puedan gozar en igualdad de condiciones, cual si fueran entenados de la sociedad, de los beneficios de la universidad, institución que, sin embargo, costea el pueblo entero, sobre sus alimentos, su ropa y su vivienda ${ }^{39}$.

Aunque en 1918 no se llegó a acuerdo sobre lo deseable que sería que la universidad fuera gratuita, lo que la dejó al margen del petitorio cordobés, pocos años después, en 1922, esta exigencia sí pasará a formar parte de las demandas que la misma Federación Universitaria de Córdoba exigirá en sus movilizaciones. Todavía hoy, vale recordar, grandes movimientos estudiantiles, como los de Chile y Colombia en 2011, tienen en este asunto uno de sus ejes fundamentales.

Sobre la segunda pista que permite rastrear esta nueva sensibilidad estudiantil, las aproximaciones del estudiantado al mundo popular, se puntualiza que en la tercera fase del movimiento, entre junio y octubre de 1918, los estudiantes recibieron muestras de apoyo por parte de organizaciones obreras que se reflejaron, entre otros aspectos, en las nutridas convocatorias que tuvieron sus manifestaciones. Y es que hubo varias concentraciones en que el número de asistentes superó con creces, a veces en más de diez veces, al total de la

estudiantado bonaerense revisar, entre otras obras, Juan Sebastián Califa, Reforma y revolución: la radicalización política del movimiento estudiantil de la UBA, 1943-1966, Buenos Aires, EUDEBA, 2014. ${ }^{39}$ Del Mazo, op. cit., 1967, 90. 
matrícula universitaria de la ciudad ${ }^{40}$. Se ha de tener presente, además, que los estudiantes también participaron de las manifestaciones promovidas por organizaciones obreras. De hecho, así como existen noticias de que Deodoro Roca, joven intelectual plenamente identificado con el movimiento estudiantil, ofició como orador en alguna de estas actividades, también hay constancia de que los estudiantes asistieron a este tipo de iniciativas. Sobre éste último punto la historiadora Victoria Chabrando informa que, en una oportunidad, los estudiantes Horacio Valdés y Enrique Barros fueron detenidos por la policía mientras participaban de una manifestación de los obreros del calzado, situación que inclusive les habría impedido formar parte del contingente estudiantil que participó en la emblemática toma de la rectoría el 9 de septiembre del citado año ${ }^{41}$.

\section{Conclusiones}

Como se ha podido comprobar, la importancia del movimiento estudiantil de Córdoba en 1918 no descansa solamente en las modificaciones sustantivas que conseguiría en el gobierno universitario o en el impacto que tendría entre el estudiantado argentino y latinoamericano. Radica, también, en que expresa una nueva sensibilidad social entre los estudiantes, una que los impulsaba a mirar más allá de las aulas y a percibir que su destino se asociaba íntimamente a los del conjunto de la población.

En una Argentina que experimentaba hondas transformaciones producto de los inicios de la industrialización y la urbanización -las cuales estaban siendo acompañadas por múltiples tensiones sociales y culturales- y en una Córdoba donde se escenificaban intensas pugnas entre quienes defendían idearios tradicionales y modernos -los cuales obedecían a su inserción parcial en los circuitos del capitalismo mundial- el estudiantado universitario comienza a multiplicarse gracias a la incorporación creciente de miembros de los emergentes sectores medios de la población y empieza a verse a sí mismo como un agente capaz de contribuir desde el plano educacional/cultural a enfrentar las dificultades que golpeaban a la sociedad. En este contexto es que se alzará para exigir que la universidad sacuda su inercia y emprenda medidas para modificar su estructura política, incrementar la calidad de la docencia e involucrarse frontalmente en la resolución de las problemáticas sociales.

Desde entonces, por tanto, el estudiantado argentino y latinoamericano comprenderá que la universidad, para ser un factor decisivo en la transformación de la realidad, debía dejar atrás el aislamiento que la caracterizaba. Esta comprensión es la que reflejan las demandas levantadas en pro de acercar la universidad al pueblo, sea en sus variantes de extensión universitaria o de universidades popular. Esta importancia es la que evidencian también algunas de las discusiones que se dieron en el marco del Primer Congreso Nacional de Estudiantes Universitarios que se realizó en Córdoba en 1918, como las relativas a los

\footnotetext{
${ }^{40}$ Sobre la masividad de las manifestaciones, esta suerte de alianza que se verifico en algunos momentos entre estudiantes y trabajadores, consultar, entre otras obras, Agüero, Ana Clarisa. Córdoba 1918, más acá de la reforma. Adrián Gorelik y Fernanda Peixoto (eds.) Ciudades sudamericanas como áreas culturales, Buenos Aires, Siglo XXI Editores, 2016, 105 y 113 y, especialmente, Portantiero, op. cit., 42, 43 y 52.

${ }^{41}$ Chabrando, op. cit., 95.
} 
alcances que éste espacio debería tener o a lo conveniente que sería que el Estado sustentara íntegramente los costos de la universidad. Y esta importancia es la que denotan también los vínculos que se dieron entre el movimiento estudiantil cordobés con los sectores populares.

Se debe hacer notar, a su vez, que las demandas estudiantiles relativas a lograr más eficiencia en el gobierno universitario y a aumentar la calidad de la docencia también poseían un cariz social que antes no evidenciaban, en el sentido que no solo respondían a ambiciones de tipo individual o gremial, sino también a inquietudes sociales. A tono con los idearios reformistas y liberales que predominaban en el país a principios del siglo XX, en el estudiantado comenzó a adquirir presencia la comprensión de que cuanto más mejorara su educación, mejor sería la educación que luego podrían proveerle a los sectores populares $^{42}$. Y se debe reparar, a su vez, que mejorar la educación de los sectores populares no solo era visto como un imperativo religioso o moral, como lo había sido desde el siglo XVI en adelante, ahora era entendido también como una condición indispensable para enfrentar con éxito las diferentes problemáticas sociales y, en último término, para progresar colectivamente.

Con todo, los estudiantes cordobeses no solo lucharon por fortalecer la autonomía de la universidad o por conseguir cambios en la docencia, es decir, por mejoras en un plano estrictamente universitario, también lo hicieron por acercar la universidad a la sociedad. Una preocupación que acompañará al estudiantado latinoamericano durante todo el siglo $\mathrm{XX}$ y que, a mediados del mismo, estará también en la base de algunas propuestas de signo socialista y revolucionario que en ese entonces proliferaron. Lo importante, por tanto, es comprender que desde fines del siglo XIX y comienzos del XX el estudiantado universitario latinoamericano comienza a adquirir una nueva sensibilidad social que se expresará en forma de reflexiones, exigencias e iniciativas que procurarán, precisamente, modificar el campo educacional conforme a estos nuevos horizontes.

\section{Referencias bibliográficas}

Agüero, Ana Clarisa. Córdoba 1918, más acá de la reforma. Adrián Gorelik y Fernanda Peixoto (eds.) Ciudades sudamericanas como áreas culturales, Buenos Aires, Siglo XXI Editores, 2016, 96-115.

Arata, Nicolás y Marcelo Mariño, La educación en la Argentina: una historia en 12 lecciones, Buenos Aires, Novedades Educativas, 2013.

\footnotetext{
${ }^{42}$ El sustrato de estas comprensiones ya había sido adelantado por otros intelectuales de renombre, como es el caso del venezolano Andrés Bello en el discurso que en 1843 realiza con motivo de asumir la rectoría de la Universidad de Chile. Véase Andrés Bello, Discurso Pronunciado en la Instalación de la Universidad de Chile el 17 de Septiembre de 1843, Pedro Grases (comp.) Antología de Andrés Bello, Caracas, Kapezlusz, 1970 [1843], 101.
} 
Bello, Andrés. Discurso Pronunciado en la Instalación de la Universidad de Chile el 17 de Septiembre de 1843, Pedro Grases (comp.) Antología de Andrés Bello, Caracas, Kapezlusz, 1970 [1843], 95-109.

Bergel, Martín. Latinoamérica desde abajo: las redes transnacionales de la Reforma Universitaria, Emir Sader y otros (comps.) La Reforma Universitaria: desafíos y perspectivas noventa años después, Buenos Aires, Consejo Latinoamericano de Ciencias Sociales, 2008, 146184.

Biagini, Hugo. La contracultura juvenil: de la emancipación a los indignados, Buenos Aires, Capital Intelectual, 2012.

Braguetto, Marco. El 'Grito de Córdoba' como hito histórico en disputa ideológica, Revista Izquierdas, $\mathrm{n}^{\circ}$ 15, Santiago de Chile, 2013, 84-103.

Buchbinder, Pablo. ¿Revolución en los claustros? La Reforma Universitaria de 1918, Buenos Aires, Sudamericana, 2008.

Buchbinder, Pablo. Historia de las universidades argentinas, Buenos Aires, Sudamericana, 2010.

Bustelo, Natalia y Domínguez Rubio, Lucas. Vitalismo libertario y Reforma Universitaria en el joven Carlos Astrada, Políticas de la memoria, ${ }^{\circ}$ 16, Buenos Aires, verano 2015/2016, 295-310.

Califa, Juan Sebastián. Reforma y revolución: la radicalización política del movimiento estudiantil de la UBA, 1943-1966, Buenos Aires, EUDEBA, 2014.

Caldelari, María y Patricia Funes, Escenas reformistas: la Reforma Universitaria 1918-1930, Buenos Aires, EUDEBA, 1998.

Cattaruzza, Alejandro. Historia de la Argentina 1916-1955, Buenos Aires, Siglo XXI Editores, 2012.

Chabrando, Victoria. El mundo ha vuelto a ponerse en movimiento en busca de nuevas fórmulas: la Reforma Universitaria en el ciclo de protestas cordobés, Córdoba, Tesis Universidad Nacional de Córdoba, 2010.

Ciria, Alberto y Horacio Sanguinetti, La Reforma Universitaria (1918-2006), Santa Fe, Universidad Nacional del Litoral, 2006.

Cornejo, Enrique. Crónica del movimiento estudiantil peruano, Juan Carlos Portantiero, Estudiantes y política en América Latina: el proceso de la Reforma Universitaria (1918-1930), Ciudad de México, Siglo XXI Editores, 1987 [1926], 232-266.

Cuevas, Yasmín y Guadalupe Olivier, Julio Antonio Mella: de líder universitario a activista social, Renate Marsiske (coord.), Movimientos estudiantiles en la historia de América Latina, volumen III, Ciudad de México, CESU/UNAM/Plaza y Valdés Editores, 2006, 105-140. 
Del Mazo, Gabriel. Estudiantes y gobierno universitario: bases doctrinarias y técnica representativa en las universidades argentinas y americanas, Buenos Aires, Librería y Editorial "El Ateneo", 1946.

Del Mazo, Gabriel (comp.) La Reforma Universitaria. Tomo I: el movimiento argentino, Lima, Universidad Nacional Mayor de San Marcos, 1967.

Fernández Retamar, Roberto. Pensamiento de Nuestra América, Buenos Aires, Consejo Latinoamericano de Ciencias Sociales, 2006.

Ferrero, Roberto. Historia crítica del movimiento estudiantil de Córdoba, Tomo 1 (1918-1943), Córdoba, Alción, 1999.

González, Julio V. Significado de la Reforma Universitaria, Dardo Cúneo (comp.) La Reforma Universitaria (1918-1930), Caracas, Biblioteca Ayacucho, 1988 [1923], 187- 206.

González Casanova, Pablo. Imperialismo y liberación: una introducción a la historia contemporánea de América Latina, Ciudad de México, Siglo XXI Editores, 1985.

Graciano, Osvaldo. Entre la torre de marfil y el compromiso político: intelectuales de izquierda en la Argentina, 1918-1955, Bernal, Editorial Universidad Nacional de Quilmes, 2008.

Haya de la Torre, Víctor Raúl. La Reforma Universitaria, Dardo Cúneo (comp.) La Reforma Universitaria (1918-1930), Caracas, Biblioteca Ayacucho, 1988 [1929], 232-240.

Klaiber, Jeffrey. The popular universities and the origins of Aprismo, 1921-1924, The Hispanic American Historical Review, Vol. 55 (4), Durham, 1975, 693-715.

Laudet, Osvaldo. Discurso del Presidente de la Federación Universitaria Argentina Osvaldo Laudet en el I Congreso Nacional de Estudiantes, Ricardo Romero (ed.) El movimiento estudiantil argentino en el siglo XX, Buenos Aires, EUDEBA, 1998 [1918], 88-90.

La Gaceta Universitaria (1918-1919), 21 números, Federación Universitaria de Córdoba, Argentina.

Las Heras, Jorge. El grito de Córdoba: la Reforma Universitaria de 1918 y su vigencia en la universidad del siglo XXI, Santiago de Chile, Sudamericana, 2009.

Mariátegui, José Carlos. La Reforma Universitaria, Emir Sader y otros (comps) La Reforma Universitaria: desafíos y perspectivas noventa años después, Buenos Aires, Consejo Latinoamericano de Ciencias Sociales, 2008 [1928], 204-212.

Markarian, Vania; María Eugenia Jung \& Isabel Wschebor, 1908 el año augural, Montevideo, Archivo General de la Universidad de La República, 2008a. 
Markarian, Vania; María Eugenia Jung \& Isabel Wschebor, 1958 El cogobierno autonómico, Montevideo, Archivo General de la Universidad de La República, 2008b.

Marsiske, Renate. Movimientos estudiantiles en América Latina: Argentina, Perú, Cuba y México, 1918-1929, Ciudad de México, CESU/UNAM, 1989.

Melgar, Ricardo. Las universidades populares en América Latina, 1910-1925, Estudios, Vol ${ }^{\circ}$ 11-12, Córdoba, 1999, 41-57.

Mella, Julio Antonio. ¿Puede ser un hecho la Reforma Universitaria? Emir Sader y otros (comps.) La Reforma Universitaria: desafíos y perspectivas noventa años después, Buenos Aires, Consejo Latinoamericano de Ciencias Sociales, 2008 [1925], 214-218.

Moraga, Fabio. Muchachos casi silvestres: la Federación de Estudiantes y el movimiento estudiantil chileno, 1906-1936, Santiago de Chile, Universidad de Chile, 2007.

Moyano, Javier. Régimen oligárquico y transformación del sistema político: el caso de los grupos gobernantes en la provincia argentina de Córdoba, 1890-1930, Ciudad de México, Tesis El Colegio de México, 2006.

Navarro, María Alejandra. Los jóvenes de la ‘ Córdoba Libre!': un proyecto de regeneración moral y cultural, Ciudad de México, Nostromo, 2009.

Orrego, Antenor. ¿Cuál es la cultura que creará América? Dardo Cúneo (comp.) La Reforma Universitaria (1918-1930), Caracas, Biblioteca Ayacucho, 1988 [1928], 255-257.

Poerner, Arthur Jose. O poder jovem: história da participação política dos estudantes brasileiros, São Paulo, Centro de Memória da Juventude, 1995 [1968].

Portantiero, Juan Carlos. Estudiantes y política en América Latina: el proceso de la Reforma Universitaria (1918-1930), Ciudad de México: Siglo XXI Editores, 1987.

Pronko, Marcela. Procesos institucionales y estructuración del movimiento estudiantil. Universidad de Luján (1979-1990), Renate Marsiske (coord.) Movimientos estudiantiles en la historia de América Latina, volumen II, Ciudad de México, CESU/UNAM/Plaza y Valdés Editores, 1999, 239-263.

Puiggrós, Adriana ¿Qué pasó en la educación argentina? Breve historia desde la conquista hasta el presente, Buenos Aires, Galerna, 2003.

Requena, Pablo. Han pasado este año cosas estupendas. Ha florecido una nueva generación: Deodoro Roca y la construcción del imaginario reformista (Córdoba 1915-1942), Córdoba: Tesis Universidad Nacional de Córdoba, 2007.

Romero, Ricardo. El movimiento estudiantil argentino en el siglo XX, Buenos Aires, EUDEBA, 1998. 
Rivas Ontiveros, José. La izquierda estudiantil en la UNAM: organizaciones, movilizaciones y liderazgos (1958-1972), Ciudad de México, Editorial Miguel Ángel Porrúa/UNAM, 2007.

Rodríguez, Fernando Diego. Un domingo en la vida de Saúl Taborda, Políticas de la memoria, $\mathrm{n}^{\circ} 16$, Buenos Aires, verano 2015/2016, 270-271.

Tcach, César. Movimiento estudiantil e intelectualidad reformista en Argentina (1918-1946), Cuadernos de Historia, 37, Santiago de Chile, 2012, 131-157.

Tedesco, Juan Carlos. Educación y sociedad en la Argentina (1880-1945), Buenos Aires, Siglo XXI Editores, 2003.

Torres Aguilar, Morelos. Cultura y revolución: la Universidad Popular Mexicana (Ciudad de México, 1912-1920), Ciudad de México, Universidad Nacional Autónoma de México, 2009.

Tünnermann, Carlos. Noventa años de la Reforma Universitaria de Córdoba (1918-2008), Buenos Aires, Consejo Latinoamericano de Ciencias Sociales, 2008.

Vallejo, Gustavo. Escenarios de la cultura científica en Argentina: ciudad y universidad (18821955), Madrid, Consejo Superior de Investigaciones Científicas, 2007.

Vera de Flasch, María. Reformas, contrarreformas y movimientos estudiantiles en la Universidad de Córdoba (1870-1936), Renate Marsiske (coord.) Movimientos estudiantiles en la historia de América Latina, volumen III, Ciudad de México, CESU/UNAM/Plaza y Valdés Editores, 2006, 21-79.

Zimmermann, Eduardo. Los liberales reformistas. La cuestión social en la Argentina, 1890-1916, Buenos Aires, Sudamericana-Universidad de San Andrés, 1995. 